\title{
Autonomous Decentralized Flow Control Allowing Control Timing Independent of Link Length
}

\author{
Chisa Takano ${ }^{\dagger}$ and Masaki Aida ${ }^{\ddagger}$ \\ $\dagger$ Graduate School of Information Sciences, Hiroshima City University, \\ 3-4-1 Ozuka-Higashi, Asa-Minami-Ku, Hiroshima, 731-3194 Japan \\ TEL: +81 82-830-1673 \\ takano@hiroshima-cu.ac.jp \\ $\ddagger$ Graduate School of System Design, Tokyo Metropolitan University, \\ 6-6 Asahigaoka, Hino-shi, Tokyo 191-0065 Japan \\ TEL: +81 42-585-8627 \\ maida@sd.tmu.ac.jp
}

\begin{abstract}
We have proposed diffusion flow control (DFC) as a technique for controlling a network, using an analogy of the diffusion phenomenon. In DFC, although each node in a network handles its local traffic flow only on the basis of the information it is aware of, the diffusion effect of DFC distributes the packet density in a network, so avoiding packet loss. In our previous work, we have assumed that each node knows the lengths of the links adjacent to it and we used control timing depending on the link length. In this paper, we describe an extension of the DFC in which the control timing is independent of the link length so allowing DFC to be used in a mobile ad hoc network.
\end{abstract}

Keywords autonomous decentralized control, diffusion equation, traffic control, wireless network

\section{Introduction}

We have previously proposed the use of autonomous decentralized technology, in which each sub-system in a network operates autonomously on the basis of only the information directly available to it, as a technique which can lead to optimal performance for the whole network. The technical reasons why such a technology is necessary are as follows.

\section{Autonomous decentralized control is suitable for high-} speed networks

In high-speed networks, since at any instant a large number of packets are in transit on links, any delay in applying control greatly affects the network performance. To address this problem, it is necessary to use time-sensitive control which operates on the basis of only partial information, without waiting to collect information from the whole network.

Autonomous decentralized control can operate under an environment where the information exchange is limited

Examples of autonomous decentralized networks are mobile ad hoc networks and P2P networks, where it is difficult to collect information about the whole network because of architectural issues. In such an environment, a control mechanism based on only partial information is needed in order to be able to act efficiently and effectively.

To solve the technical problem concerning high-speed networks, we have proposed the use of diffusion type flow control (DFC) [1], in which each node in a network acts autonomously on the basis only of local information but the cumulative effect of the local decision-making at each node leads to high performance of the network as a whole. In addition, we have investigated aspects of network performance of DFC for high-speed networks [2, 3, 4, 5]. DFC is a flow control which determines the transmission rate based only on information it is aware of. DFC allows packet loss due to buffer overflow to be avoided by ensuring that the density of packets stored in each node is not unevenly distributed in certain nodes, but is rapidly diffused over the whole network. The diffusion effect associated with DFC leads to steady data transmission in even high-speed networks. Moreover, the cost of the network can be reduced because the capacity of the storage buffers in the nodes can be reduced.

In this paper, we consider the solution of the technical issue in the mobile ad hoc network, where the link lengths are 


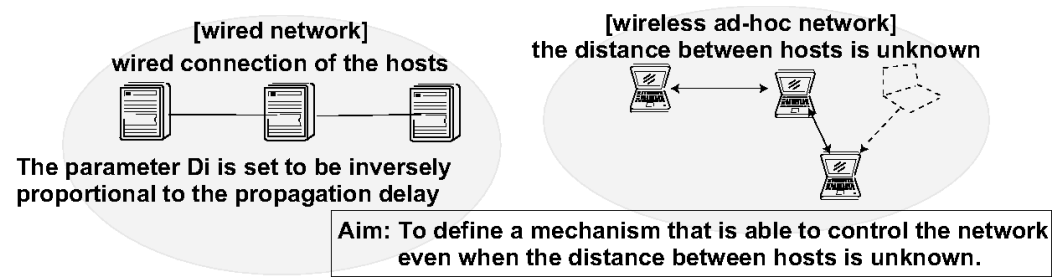

Figure 1. Application of the DFC to the wireless ad hock network

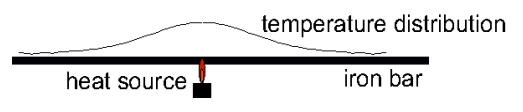

Figure 2. Example of thermal diffusion phenomena.

not known, so as to establish how autonomous decentralized control under the environment where the information exchange is even more limited than the cases considered before.

A mobile ad hoc network is an autonomous decentralized network and is composed of wireless systems having a relay function. For ad hoc network technology, many techniques have been introduced concerning, especially, the control of route selection [7, 8]. Routing algorithms that take account of security, low power consumption, and Quality of Service (QoS) have been proposed in [9, 10]. In addition to routing algorithms, scheduling algorithms aimed at improving the efficiency of networks offering controlled QoS have been described [11, 12].

In this paper, we apply the framework of DFC, which has been proposed for the solution of the high-speed network issues, to mobile ad hoc networks, and investigate the realizability of DFC in networks where information exchange is fundamentally and structurally limited. In the DFC implementations described in the past, each node had to know the value of the propagation delay of the links to the adjacent nodes. However, in a mobile ad hoc network it is difficult to know the propagation delay in real time because the distance to the other hosts is continually changing. Therefore, a control technique that requires knowledge of the link delay to adjacent hosts is not useful (Fig. 1). In this paper, we extend DFC so that it can be applied even to a wireless environment and establish a control method that doesn't depend on the link length.

The remainder of this paper is organized as follows. Sec. 2 describes the basic operation of the previously described type of DFC in wired networks. In Sec. 3 we propose a new type of DFC in which the control timing interval, that is the time interval at which flow control is applied, is independent of the link length. Conditions for simulating this new technique are described in Sec. 4 and the simulation results are given in Sec. 5. Finally, conclusions are made in Sec. 6.

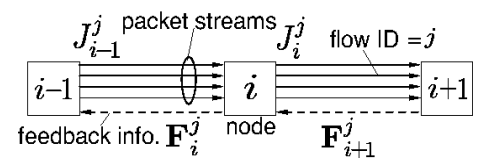

Figure 3. Node interactions in our flow control model.

\section{Diffusion-Type Flow Control Mechanism}

In this section, we describe the framework of DFC as applied to high-speed wired networks.

\subsection{Aim of Diffusion-Type Flow Control Mecha- nism}

The most remarkable characteristic of DFC is that it provides a framework in which the implementation of local decision-making at each node leads to high and stable performance for the whole network. To explain the basis of this mechanism, we show the principle of this flow control model through the following analogy [3].

When we heat a point on a cold iron bar, the temperature distribution follows a normal distribution and heat spreads through the whole bar by diffusion (Fig. 2). In this process, the action in a minute segment of the iron bar is very simple: heat flows from the hotter side towards the cooler side. The rate of heat flow is proportional to the temperature gradient. Although each segment acts autonomously, based on its local information, the temperature distribution of the whole iron bar exhibits orderly behavior. In DFC in a network, each node controls its local packet flow to an adjacent node, so that it is proportional to the difference between the number of packets in that node and the number in the adjacent node. The overall effect is that the distribution of the total number of packets in each node in the network becomes uniform over time.

\subsection{Behavior of Diffusion-Type Flow Control Mechanism}

Figure 3 shows the interactions between nodes (routers) in DFC, using a network model with a simple 1-dimensional configuration. In DFC, node $i(i=1,2, \ldots)$ transfers 
packets to node $i+1$, and node $i+1$ sends feedback information $\mathbf{F}_{i+1}$ to node $i$. When node $i$ receives feedback information from downstream node $i+1$, it determines the transmission rate for packets to the downstream node $i+1$ using the received feedback information, and it adjusts its transmission rate towards the downstream node $i+1$. Let us assume that there are $M_{i}$ flows sharing the link between node $i$ and node $i+1$, and they are identified by $j\left(j=1,2, \ldots, M_{i}\right)$. Each node $i$ autonomously determines the transmission rate $J_{i}^{j}$ for flow $j$ on the basis of the feedback information obtained from the downstream node $i+1$ and its own information. The transmission of packets and feedback information both experience the same propagation delay.

The transmission rate $J_{i}^{j}(t)$ for flow $j$ of node $i$ at time $t$ is determined by

$$
\begin{aligned}
J_{i}^{j}(t) & =\max \left(0, \min \left(L_{i}^{j}(t), \tilde{J}_{i}^{j}(t)\right)\right), \\
\tilde{J}_{i}^{j}(t) & =r_{i}^{j}\left(t-d_{i}\right)-D_{i}\left(n_{i+1}^{j}\left(t-d_{i}\right)-n_{i}^{j}(t)\right),
\end{aligned}
$$

where $L_{i}^{j}(t)$ denotes the available bandwidth for flow $j$ of the link from node $i$ to node $i+1$ at time $t, n_{i}^{j}(t)$ denotes the number of packets belonging to flow $j$ in node $i$ at time $t, r_{i}^{j}\left(t-d_{i}\right)$ is the required rate for flow $j$ derived from the feedback information from the downstream node $i+$ 1 (hereafter called the "notified rate"), and $d_{i}$ denotes the propagation delay between nodes $i$ and $i+1$. $D_{i}$ is the parameter used by DFC.

Let the bandwidth of the link from node $i$ to node $i+1$ be $B_{i}$, and $L_{i}^{j}(t)$, the available bandwidth for flow $j$, is derived by assuming that the bandwidth $B_{i}$ is shared by the different flows according to a weight $\tilde{J}_{i}^{j}(t)[4]$, that is,

$$
L_{i}^{j}(t)=B_{i} \times \frac{\tilde{J}_{i}^{j}(t)}{\sum_{k=1}^{M_{i}} 1_{\{k=\text { active }\}} \times \tilde{J}_{i}^{k}(t)},
$$

where $1_{\{k=\text { active }\}}$ is the indicator function which is equal to 1 if flow $k$ is active at time $t$, and otherwise is equal to 0 .

The feedback information for flow $j$ created at fixed intervals $\tau_{i}$ by node $i$ consists of three quantities, as follows:

$$
\mathbf{F}_{i}^{j}(t)=\left(r_{i-1}^{j}(t), n_{i}^{j}(t), \ell_{i}^{j}(t)\right) .
$$

For DFC applied to a wired network, we assumed that the control timing interval is equal to the propagation delay $\left(\tau_{i}=d_{i-1}\right) \cdot \ell_{i}^{j}(t)$ is the information defining the maximum available bandwidth. $\ell_{i}^{j}(t)$ and the notified rate $r_{i-1}^{j}(t)$ are limited by the available bandwidth $L_{i}^{j}$ as follows.

$$
\begin{aligned}
\ell_{i}^{j}(t) & =\min \left(L_{i}^{j}(t), \ell_{i+1}^{j}(t)\right), \\
r_{i-1}^{j}(t) & =\max \left(0, \min \left(L_{i}^{j}(t)\right), \tilde{J}_{i}^{j}(t)\right) .
\end{aligned}
$$

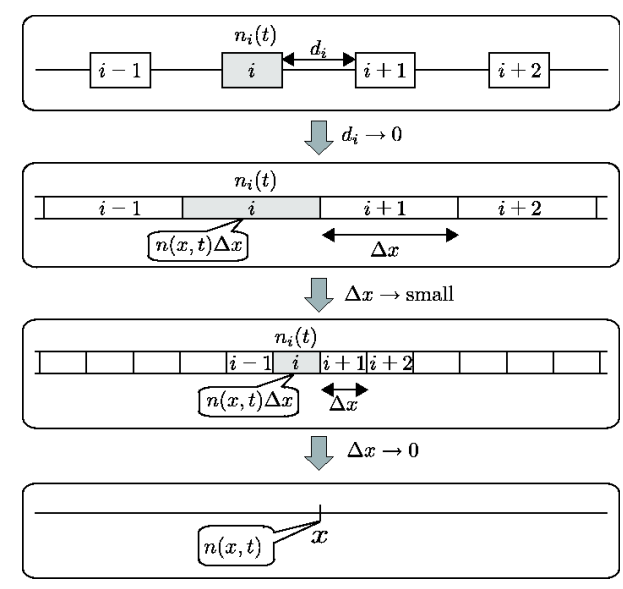

\section{Figure 4. Correspondence between discrete and continuous network models.}

Parameter $D_{i}$ is chosen to be inversely proportional to the propagation delay as follows:

$$
D_{i}=\frac{D}{d_{i}}
$$

where $D(>0)$, which is a positive constant, is the diffusion coefficient. As shown in [2], setting parameter $D_{i}$ in accordance with Eq.(7) allows the complexity of the network model to be absorbed, and provides high performance and stability even if the configuration of the network becomes complex.

\section{Extension of DFC to allow the control tim- ing to be independent of the link propaga- tion delay}

Although we assumed $d_{i}=\tau_{i+1}$ in the previous section, the two parameters have different origins, and so we can regard them as different (independent) items. In this section, we show that, for more general applicability, parameter $D_{i}$ should be determined by using the control timing inverval $\tau_{i}$ (not the propagation delay di) as follows.

$$
D_{i}=\frac{D}{\tau_{i+1}}
$$

Here, the interval of DFC's actions $\Delta t\left(=\tau_{i}\right)$ is unchanged. $\Delta t$ is independent of $d_{i}$. To introduce a single discrete space reflecting an entire 1-dimensional network structure, we divide the continuous 1-dimensional space into lengths of $\Delta x$ which are also independent of $d_{i}$.

Next, we consider the continuous values, $\tilde{J}^{j}(x, t)$, $r^{j}(x, t)$ and $n^{j}(x, t)$ as continuous approximations of $\tilde{J}_{i}^{j}(t), r_{i}^{j}(t)$ and $n_{i}^{j}(t)$ in order to confirm the relationship between the discrete space and the continuous space. If 


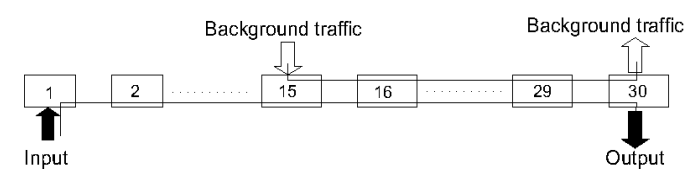

Figure 5. Simulation Model.

$n^{j}(x, t)$ is the packet density at time $t$ in discrete space $x$, we obtain the following relation:

$$
n_{i}^{j}(t)=n^{j}(x, t) \Delta x+o(\Delta x) .
$$

To appropriately associate the number of packets $n_{i}^{j}(t)$ in a discrete space with the packet density $n^{j}(x, t)$ in a continuous space, Eq.(2) needs to be modified for the continuous case, and may be approximated by the following equation .

$$
\tilde{J}^{j}(x, t)=r^{j}(x, t)-\kappa \frac{\partial n^{j}(x, t)}{\partial x},
$$

where $\kappa$ is a diffusion coefficient in the physical diffusion phenomenon (in the continuous space).

The relationship between the discrete space and the continuous space is shown in Fig. 4. First, we take the limit of the distance between the adjacent nodes as $d_{i} \rightarrow 0$. The second panel in Fig. 4 shows this situation. Here, the interval of DFC's actions, $\Delta t$ is unchanged. Next, taking the limit of $\Delta x$ to be 0 , we obtain a continuous approximation of the 1-dimensional network. Note that $\Delta t$ should approach 0 concurrently with $\Delta x \rightarrow 0$. The relationship between the packet density $n(x, t)$ and the number of packets stored in a node $n_{i}(t)$ is also shown in Fig. 4.

After taking the limit as $d_{i} \rightarrow 0$, we compare the second term on the right hand side of (2) with that of (9) and get the following relation.

$$
\begin{aligned}
\kappa \frac{\partial n^{j}(x, t)}{\partial x} & =\kappa \lim _{\Delta x \rightarrow 0} \frac{n^{j}(x+\Delta x, t)-n^{j}(x, t)}{\Delta x} \\
& =\kappa \lim _{\Delta x \rightarrow 0} \frac{n_{i+1}^{j}(t)-n_{i}^{j}(t)}{(\Delta x)^{2}} \\
& =\kappa \lim _{\Delta x \rightarrow 0} \frac{\Delta t}{(\Delta x)^{2}} \frac{n_{i+1}^{j}(t)-n_{i}^{j}(t)}{\Delta t} .
\end{aligned}
$$

We have Eq. (8) by the relation $D=\kappa \Delta t /(\Delta x)^{2}$ which is already known from [5].

\section{Simulation}

By means of simulation, we show that setting the parameter Di in accordance with Eq. (8) leads to the diffusion effect of DFC, even if information about the propagation delay is unknown. For the performance comparison, we set the value of the parameter $D_{i}$ in inverse proportion to the propagation delay. Simulations were made by using ns2 simulator.
Figure 5 shows the network model used in the simulation, with 30 mobile hosts. Although this 1-dimensional model looks simple, it represents a part of a network and describes a path of the target end-to-end flow extracted from the whole network. Each packet has a fixed length of 1,500 Bytes and the link bandwidth is 4, 400 packets/s which corresponds to a link bandwidth of 54 Mbps.

The simulations described here assume a non-uniform network configuration, that is, the lengths of the links are different. In this model, the propagation delay $d_{i}$ is specified by a probability distribution with the mean $d=0.5$ $\mu \mathrm{sec}$, which corresponds to a mean link length of about 100 $\mathrm{m}$. The propagation delay of each link is determined in advance. The delay consists of a fixed component of 0.25 $\mu$ sec and a variable component that obeys an exponential distribution with a mean of $0.25 \mu \mathrm{sec}$. $D$ is equal to 0.4 . The simulation scenario is as follows. There are two UDP flows (the target and background flows). The target flow is between host 1 and host 30, while the background flow is between host 15 and host 30 . The target flow and the background flow start at simulation time $t=0 \mathrm{sec}$ and $t=1 \mathrm{sec}$, respectively. Both flows have greedy traffic, that is, the rate of each flow is as large as possible. For each simulation, the values of the control timing interval of all hosts are the same $(5,10,50,100,500$ or $1,000 \mathrm{msec})$. Parameter $D_{i}$ is set by Eq.(8) or the following scheme for the performance comparison.

$$
D_{i}=\frac{D}{d_{i}} \times\left(\frac{d}{\tau_{i+1}}\right)
$$

This denotes that parameter $D_{i}$ is set in inverse proportion to the propagation delay.

The object of this paper is the determination of parameter $D_{i}$ independent of link length. We have discussed previously how the network performance is improved compared with other methods in [1, 2, 3, 4].

\section{Simulation Results}

We investigate the effectiveness of setting $D_{i}$ as described in Eq. (8). Figure 6 shows the results for the behavior of the packet distribution among individual hosts for DFC when $D_{i}$ is set as in Eq. (8). The different rows in Fig. 6 show the results for DFC having a control timing interval $\tau=5,10,50,100,500$ and $1,000 \mathrm{msec}$, respectively, while the different panels refer to different times after the start of the simulation. The horizontal axis of each graph denotes the host ID, the vertical axis denotes the number of packets stored in the host, and simulation time ( $\mathrm{sec}$ ) is shown in each graph. It can be seen that once the background traffic starts entering the simulation network (after $1 \mathrm{~s})$, DFC control at each host in the network prevents the number of stored packets building up at a certain host. We 

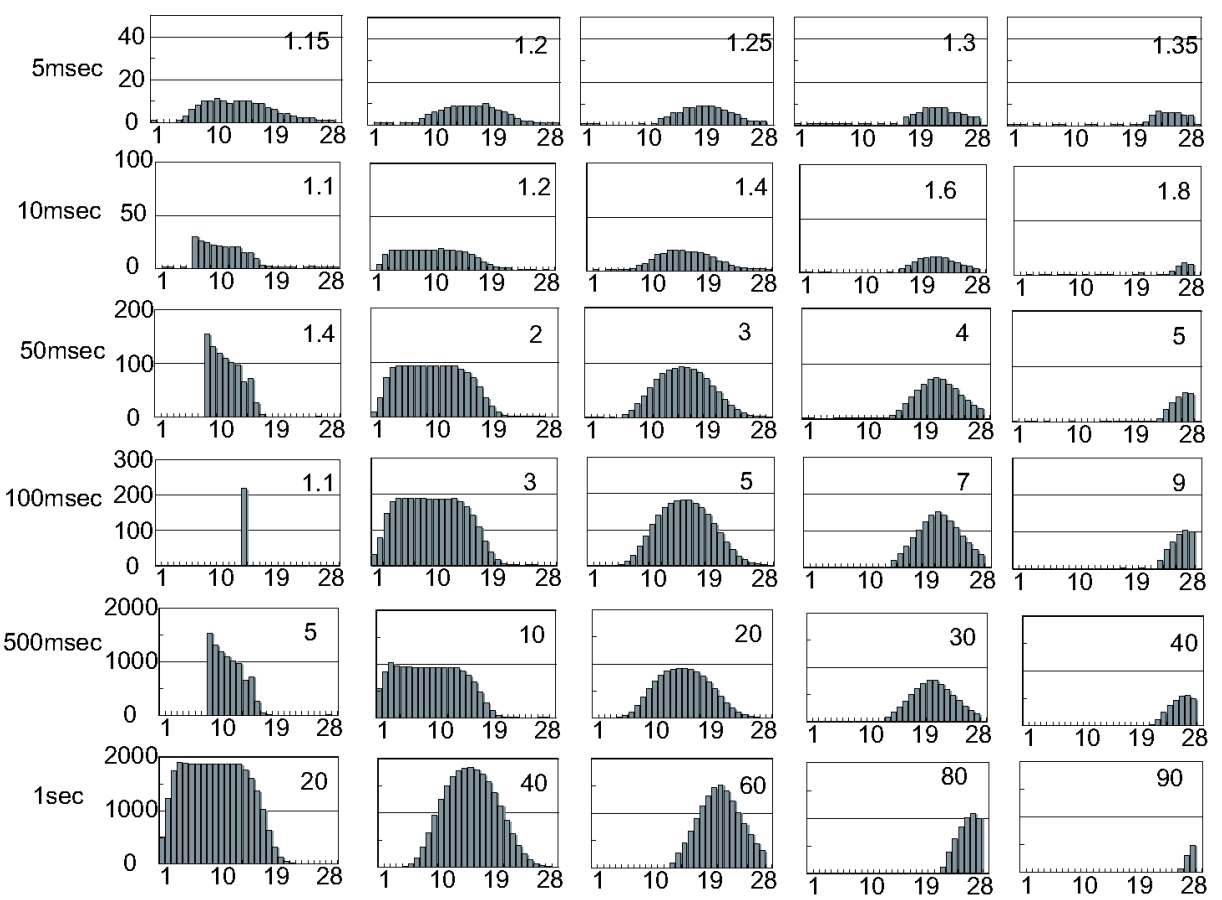

Figure 6. Temporal evolution of distribution of packets stored in each host. ( $D_{i}$ is inversely proportional to $\tau$ )

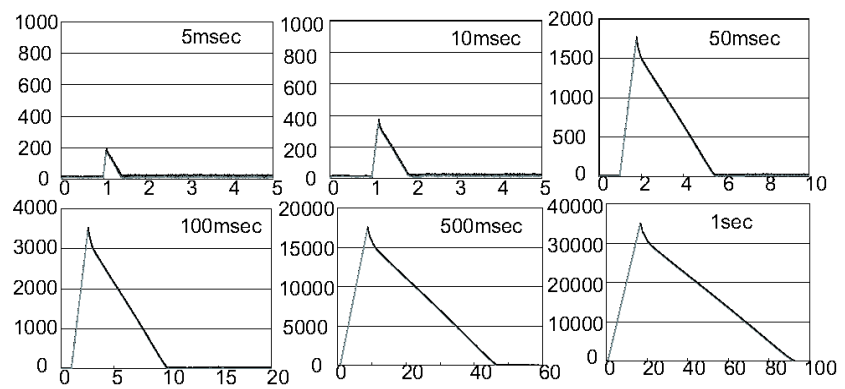

Figure 7. Temporal evolution of the total number of packets that are stored in hosts, for different values of control timing interval. $\left(D_{i}\right.$ is inversely proportional to $\tau$ )

can see from Fig. 6 that as the control timing interval increases, the number of packets (the value of the vertical axis) stored in the hosts increases, because the effectiveness of DFC works more slowly through the whole network. In all the results, regardless of the control timing interval, the packets do not become concentrated at the potentially congested host 15 and the number of packets stored in the hosts becomes smoothly distributed over the network and decreases with time.

Panels in Fig. 7 show the temporal evolution of the total number of packets stored in hosts, when the values of the control timing interval $\tau$ are equal to $5,10,50,100,500$ and $1000 \mathrm{msec}$, respectively. The horizontal axis denotes

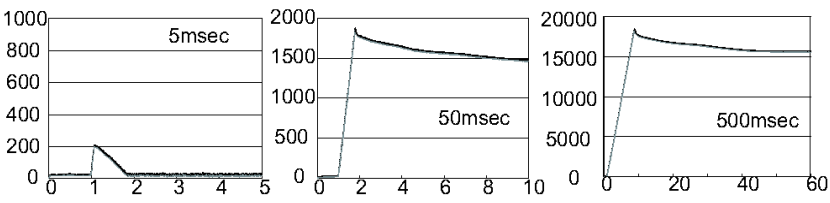

Figure 9. Temporal evolution of the total number of packets that are stored in hosts. ( $D_{i}$ is inversely proportional to $d_{i}$ )

the simulation time ( $\mathrm{sec}$ ) and the vertical axis denotes the total number of packets that are stored in hosts, and the control timing interval is shown on each graph. These results also show that after the total number of total packets initially grows once congestion starts at $t=1 \mathrm{sec}$, the total number of packets then decreases almost linearly with time and approaches 0 as a result of the diffusion effect of DFC. Moreover, the maximum value of the total number of packets stored in hosts increases in proportion to the control timing interval $\tau$ and the elapsed time until the total number of packets returns to 0 is larger in proportion to the control timing interval.

Next, Figs. 8 and 9 show the results when $D_{i}$ is set to be inversely proportional to the propagation delay. Each panel in Fig. 8 shows the distribution of the number of packets stored in the host in case of the control timing interval $\tau=5,50$ or $500 \mathrm{msec}$. In this case, the distribution of the packets is not smoothly diffused, as can be seen when compared with the results (Fig. 6) for the case that $D_{i}$ was 

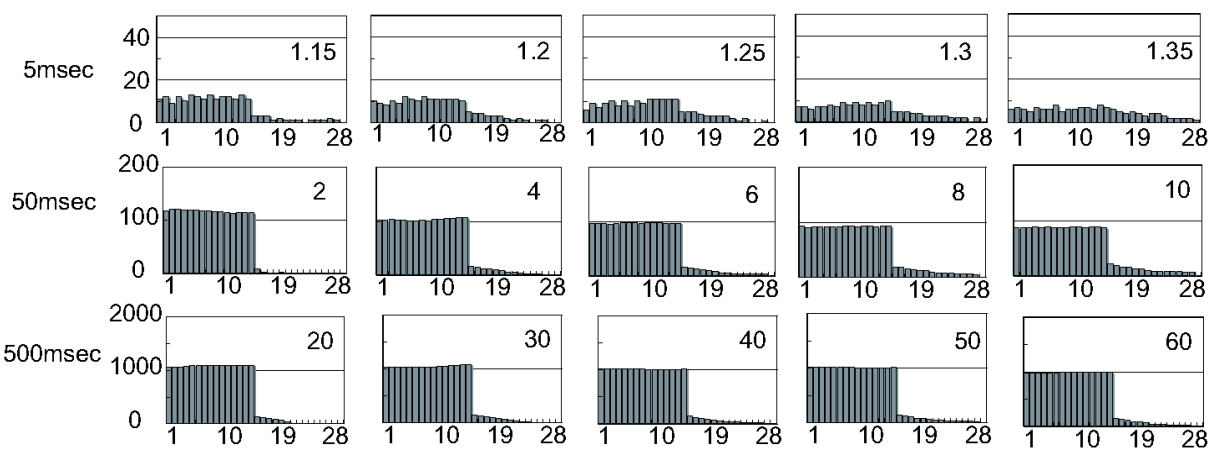

Figure 8. Temporal evolution of distribution of packets stored in each host. ( $D_{i}$ is inversely proportional to $d_{i}$ )

inversely proportional to $\tau$. Figure 9 shows the temporal evolution of the total number of packets stored. Compared with Fig. 7, the time interval until the total number of packets converges to 0 is very large. This means that cooperative action between hosts doesn't perform well in this case and the diffusion effect of DFC is not realized.

\section{Concluding Remarks}

In this paper, our target is the application of DFC to a mobile ad hoc network, and the extension of DFC to situations where the control timing is independent of link length. Our results have showed that the diffusion effect of DFC is achieved when the parameter $D_{i}$ is inversely proportional to the control timing interval even if the propagation delay is unknown. Future work will include verifying the performance of DFC by using a network model whee the distance between each host changes dynamically.

\section{Acknowledgement}

This research was partially supported by the National Institute of Information and Communications Technology (NiCT) and the Grant-in-Aid for Scientific Research (S) No. 18100001 (2006-2010) from the Japan Society for the Promotion of Science.

\section{References}

[1] C. Takano and M. Aida, "Stability and adaptability of autonomous decentralized flow control in high-speed networks," IEICE Transactions on Communications, vol.E86-B, no.10, pp.2882-2890, 2003.

[2] C. Takano, M. Aida, and S. Kuribayashi, "Autonomous decentralized flow control in high-speed networks with inhomogeneous configurations," IEICE Transactions on Communications, vol.E87-B, no.6, pp.1551-1560, 2004.
[3] C. Takano and M. Aida, "Diffusion-type autonomous decentralized flow control for end-to-end flow in highspeed networks," IEICE Transactions on Communications, vol.E88-B, no.4, pp.1559-1567, 2005.

[4] C. Takano and M. Aida, "Diffusion-type autonomous decentralized flow control for multiple flows," IEICE Transactions on Communications, vol. E90-B, no. 1, pp. 21-30, January 2007.

[5] C. Takano, K. Sugiyama and M. Aida, "Parameter Design for Diffusion-Type Autonomous Decentralized Flow Control," IEICE Transactions on Communications, vol. E91-B, no. 9, pp. 2828-2837, September 2008.

[6] E. Royer and C.-K. Toh, "A review of current routing protocols for ad hoc wireless networks," IEEE Personal Communications, pp. 46-55, April 1999.

[7] E. Perkins, M. Belding-Royer and R. Das, "Ad hoc ondemand distance vector (AODV) routing," Request for Comments 3561, July 2003.

[8] T. Clausen and P. Jacquet, "Optimized link state routing protocol (OLSR)," Request for Comments 3626, October 2003.

[9] Y. Hu, A. Perrig, and B. Johnson, "Ariadne: A Secure on-demand routing protocol for ad hoc networks," in Proceedings of ACM MobiCom 2002, Sept. 2002.

[10] Y. Xu, J. Heidemann and D. Estrin, "Geographyinformed energy conservation for ad hoc routing," in Proceedings of ACM Mobile Computing and Networking 2001, July 2001.

[11] X. Lin and N. B. Shro, "Joint rate control and scheduling in multihop wireless networks," in Proceedings of IEEE Conference on Decision and Control, Bahamas, 2004.

[12] L. Bui, A. Eryilmaz and R. Srikant, "Joint Asynchronous Congestion Control and Distributed Scheduling for Multi-Hop Wireless Networks", in Proceedings of IEEE INFOCOM 2006, Apr. 2006. 